\title{
METACOMPACTNESS, PARACOMPACTNESS, AND INTERIOR-PRESERVING OPEN COVERS
}

\author{
BY
}

\author{
HEIKKI J. K. JUNNILA ${ }^{1}$
}

\begin{abstract}
In this paper metacompactness and paracompactness are characterized in terms of the existence of closure-preserving closed refinements and interior-preserving open star-refinements of interior-preserving directed open covers of a topological space. Several earlier results on metacompact spaces and paracompact spaces are obtained as corollaries to these characterizations. For a Tychonoff-space $X$, metacompactness of $X$ is characterized in terms of orthocompactness of $X \times \beta X$.
\end{abstract}

1. Introduction. Closure-preserving closed covers have found many applications in general topology, after they were used by E. Michael to give an important characterization of paracompactness [13]. In the following, we show that besides closure-preserving closed covers, interior-preserving open covers also play a fundamental role in the study of covering properties of topological spaces. The covering properties that we consider in this paper are metacompactness and paracompactness; the ideas used here can, however, also be applied to study other properties, such as subparacompactness and $\theta$-refinability, as we intend to show in a forthcoming paper.

The main results of this paper are the following: (i) a topological space is metacompact if, and only if, every interior-preserving directed open cover of the space has a closure-preserving closed refinement; (ii) a topological space is paracompact if, and only if, every interior-preserving directed open cover of the space has a $\sigma$-closure-preserving closed refinement by sets whose interiors cover the space. To arrive at these results, we need to use two rather dissimilar types of characterizations of covering properties. The first kind of a characterization is exemplified by Alexandroff's and Urysohn's result that a topological space is compact if, and only if, every monotone open cover of the space has a finite subcover [1]; the best-known example of a characterization of the other kind is A. H. Stone's result that paracompactness and full normality are equivalent properties in the class of Hausdorff spaces [19].

Received by the editors December 30, 1977.

AMS (MOS) subject classifications (1970). Primary 54D20.

Key words and phrases. Metacompact, paracompact, interior-preserving, closure-preserving, directed cover, orthocompact, $M M^{\#}$-space.

'The results of this paper were obtained in 1974 while the author was studying for the Licenciate degree at the University of Helsinki. 
2. Preliminaries. For the meaning of concepts used without definition in this paper, see [6]; note, however, that we do not require paracompact and metacompact spaces to satisfy any separation axioms and that in our terminology, regular spaces are not necessarily $T_{1}$.

Throughout the following, $X$ denotes a topological space. An open cover $\mathcal{U}$ of $X$ is interior-preserving if $\cap \mathcal{U}^{\prime}$ is open for each $\mathcal{U}^{\prime} \subset \mathcal{U}$ or, equivalently, if the closed family $\{X \sim U \mid U \in \mathcal{U}\}$ is closure-preserving. For some properties of interior-preserving families, see [10]. Note that every point-finite open cover is interior-preserving. Well-monotone open covers constitute another important class of interior-preserving open covers. A family $\mathcal{E}$ of sets is monotone if the relation $\subset$ of set inclusion is a linear order on $\mathcal{E}$; if this order is a well-order, then we say that $\mathcal{L}$ is well-monotone. If $\mathcal{L}$ is well-monotone and $\mathcal{L}^{\prime} \subset \mathcal{L}$, then the set $\cap \mathcal{L}^{\prime}$ belongs to $\mathcal{L}^{\prime}$; hence we see that every well-monotone open cover of $X$ is interior-preserving.

In light of the preceding observation, the following result makes it possible to restrict one's attention to refinements of interior-preserving open covers when considering metacompact and paracompact spaces. The part of the following theorem dealing with metacompactness was announced in [16]; however, since no proof of it has appeared in the literature, we include one here.

THEOREM 2.1 ([16], [12]). A topological space is metacompact (paracompact) iff every well-monotone open cover of the space has a point-finite (locally finite) open refinement.

Proof. Necessity is obvious. To prove sufficiency, assume that every well-monotone open cover of $X$ has a point-finite (locally finite) open refinement. For each cardinal number $\mathfrak{f}$, denote by $P(\mathfrak{f})$ the following proposition: if $\mathscr{U}$ is an open cover of $X$ with $|\mathscr{U}|=\mathfrak{f}$, then $\mathscr{U}$ has a point-finite (locally finite) open refinement. We use transfinite induction to show that $P(\mathfrak{f})$ holds for every cardinal $\mathfrak{f}$. For $\mathfrak{f}$ finite, $P(\mathfrak{f})$ is trivially true. Let $\mathfrak{f}$ be an infinite cardinal such that $P\left(\mathfrak{f}^{\prime}\right)$ holds for every $\mathfrak{f}^{\prime}<\mathfrak{f}$. To show that $P(\mathfrak{f})$ holds, let $\mathscr{U}$ be an open cover of $X$ with $|\mathscr{}|=\mathfrak{f}$. We can represent $\mathscr{U}$ in the form $\mathscr{U}=\left\{U_{\alpha} \mid \alpha<\gamma\right\}$, where $\gamma$ is the initial ordinal corresponding to f. For each $\alpha<\gamma$, let $V_{\alpha}=\cup_{\beta<\alpha} U_{\beta}$. Then the family $\mathscr{V}=\left\{V_{\alpha} \mid \alpha<\gamma\right\}$ is a well-monotone open cover of $X$. Let $\mathscr{W}$ be a point-finite (locally finite) open refinement of $\mathcal{V}$. For each $W \in \mathcal{W}$, let $\alpha(W)<\gamma$ be such that $W \subset V_{\alpha(W)}$. For each $\alpha<\gamma$, let $P_{\alpha}=\cup\{W \in \mathscr{W} \mid \alpha(W)>\alpha\}$ and let $\mathscr{P}_{\alpha}=\left\{P_{\alpha}\right\} \cup\left\{U_{\beta} \mid \beta \leqslant \alpha\right\}$. For each $\alpha<\gamma$, the family $\mathscr{P}_{\alpha}$ is an open cover of $X$ and $\left|\mathscr{P}_{\alpha}\right|<\mathfrak{f}$; hence, by the induction assumption, there exists a point-finite (locally finite) open cover $\mathscr{Q}_{\alpha}$ of $X$ such that $\mathscr{Q}_{\alpha}$ refines $\mathscr{P}_{\alpha}$. For each $W \in \mathcal{Q}$, let $R(W)=\left\{W \cap Q \mid Q \in \mathscr{Q}_{\alpha(W)}\right.$ and $Q \subset U_{\alpha}$ for some 
$\alpha \leqslant \alpha(W)\}$. It is easily seen that the family $\Re=\cup\{\Re(W) \mid W \in \mathscr{W}\}$ is point-finite (locally finite) and open and that for each $R \in \mathcal{R}$, we have $R \subset U$ for some $U \in \mathcal{Q}$. To show that $\Re$ is a cover, let $x \in X$. Since the family $(\mathscr{W})_{x}=\{W \in \mathscr{W} \mid x \in W\}$ is finite, there exists $W \in(\mathscr{W})_{x}$ such that $\alpha\left(W^{\prime}\right) \leqslant \alpha(W)$ for every $W^{\prime} \in(W)_{x}$. Let $\beta=\alpha(W)$ and let $Q \in \mathcal{Q}_{\beta}$ be such that $x \in Q$. We have $x \notin P_{\beta}$ and it follows, since $\mathscr{2}_{\beta}$ refines $\mathscr{P}_{\beta}$, that $Q \subset U_{\alpha}$ for some $\alpha \leqslant \beta$; hence $W \cap Q \in \Re(W)$. Since $x \in W \cap Q$ and $\Re(W) \subset \Re$, we have $x \in \cup \Re$. We have shown that $\Re$ covers $X$ and it follows that $\mathcal{R}$ is a refinement of $\mathscr{U}$. We have shown that $P(\mathfrak{f})$ holds. This completes the induction and the proof.

Let $\mathcal{L}$ and $\Re$ be families of subsets of $X$. For each $A \subset X$, we let $(\mathscr{T})_{A}=\{N \in \mathscr{T} \mid N \cap A \neq \varnothing\}$; if $A=\{x\}$, then we write $(\mathscr{T})_{x}$ in place of $(\Re)_{A}$. We say that $\Re$ is a partial refinement of $\mathcal{L}$ if for each $N \in \mathfrak{R}$, we have $N \subset L$ for some $L \in \mathcal{E}$. We say that $\Re$ is a pointwise (local) $W$ refinement of $\mathcal{L}$ if $\cup \mathscr{T}=\cup \mathcal{L}$ and for each $x \in X$, the family $(\mathscr{T})_{x}$ (for some neighborhood $U$ of $x$, the family $(\mathcal{U})_{U}$ ) is a partial refinement of some finite subfamily $\mathcal{L}^{\prime}$ of $\mathcal{L}$; a pointwise (local) star-refinement is defined similarly except that now we require that $\mathcal{L}^{\prime}=\{L\}$ for some $L \in \mathcal{L}$.

The concept of a pointwise $W$-refinement was introduced by $\mathrm{J}$. M. Worrell, Jr. in his study of metacompact spaces ([22] and [23]). This concept provides a simultaneous generalization of the concepts of a pointwise star-refinement and a point-finite refinement; hence the following result, essentially due to Worrell [22], is of utmost importance in the study of covering properties.

Proposition 2.2. Let $\left\langle\mathcal{Q}_{n}\right\rangle$ be a sequence of open covers of $X$ such that for each $n \in \mathbf{N}, \mathscr{Q}_{n+1}$ is a pointwise $W$-refinement of $\mathscr{Q}_{n}$. Then $\mathscr{U}_{1}$ has an open refinement $\mathfrak{V}=\cup_{n=2}^{\infty} \mathfrak{V}_{n}$ such that for all $n \in \mathbf{N}$ and $K \subset X$, if $\left(\mathcal{U}_{n+1}\right)_{K}$ is a partial refinement of a subfamily $\mathcal{U}^{\prime}$ of $\mathcal{U}_{n}$, then $\left|\left(\mathcal{V}_{n+1}\right)_{K}\right| \leqslant\left|\mathcal{U}^{\prime}\right|$.

Proof. Represent $\mathscr{U}_{1}$ in the form $\mathscr{U}_{1}=\left\{U_{\alpha} \mid \alpha<\gamma\right\}$, where $\gamma$ is some ordinal number. For each $U \in \cup_{n=1}^{\infty} \mathscr{Q}_{n}$, denote by $\alpha(U)$ the least ordinal $\alpha<\gamma$ such that $U \subset U_{\alpha}$; note that if $U^{\prime} \in \cup_{n=1}^{\infty} \mathscr{Q}_{n}$ is such that $U \subset U^{\prime}$, then $\alpha\left(U^{\prime}\right) \geqslant \alpha(U)$. For $n>1$, we say that the family $U_{n-1}$ is precise at a set $U \in \mathscr{U}_{n}$, if $\alpha\left(U^{\prime}\right)=\alpha(U)$ whenever $U \subset U^{\prime} \in \mathscr{U}_{n-1}$, and we let $\mathscr{W}_{n}=$ $\left\{U \in \mathscr{Q}_{n} \mid \mathscr{Q}_{n-1}\right.$ is precise at $\left.U\right\}$.

We show that the family $\cup_{n=2}^{\infty} \mathscr{W}_{n}$ covers $X$. Let $x \in X$. For every $n>1$, denote by $\alpha_{n}$ the least upper bound of the set $\left\{\alpha(U) \mid U \in\left(\mathscr{Q}_{n}\right)_{x}\right\}$ and note that we have $\alpha_{n}<\gamma$, since $\mathscr{U}_{n}$ is a pointwise $W$-refinement of $\mathscr{U}_{1}$. For every $n>1$, we have $\alpha_{n+1} \leqslant \alpha_{n}$, because $\mathcal{U}_{n+1}$ is a refinement of $\mathcal{U}_{n}$. It follows that there exist $\beta<\gamma$ and $k \geqslant 3$ such that $\alpha_{n}=\beta$ for every $n \geqslant k-1$. Since $\mathcal{U}_{k+1}$ is a pointwise $W$-refinement of $\mathcal{U}_{k}$, there is a finite family $\mathcal{U} \subset\left(\mathcal{U}_{k}\right)_{x}$ such that the family $\left(\mathcal{U}_{k+1}\right)_{x}$ is a partial refinement of the family $\mathscr{U}$. Let 
$U \in \mathcal{Q}$ be such that $\alpha\left(U^{\prime}\right) \leqslant \alpha(U)$ for every $U^{\prime} \in \mathcal{U}$. Since $\left(\mathcal{U}_{k+1}\right)_{x}$ is a partial refinement of $\mathcal{U}$, we have $\alpha_{k+1} \leqslant \alpha(U)$ and, on the other hand, since $U \in \mathcal{Q}_{k}$, we have $\alpha(U) \leqslant \alpha_{k}$. Since $\alpha_{k+1}=\alpha_{k}=\beta$, it follows that $\alpha(U)=$ $\beta$. Now since $\alpha_{k-1}=\beta$, we see that $\mathscr{U}_{k-1}$ is precise at $U$ : for every $U^{\prime} \in\left(\vartheta_{k-1}\right)_{x}$, we have $\alpha\left(U^{\prime}\right) \leqslant \beta$, that is, $\alpha\left(U^{\prime}\right) \leqslant \alpha(U)$, and if $U \subset U^{\prime}$, then we also have $\alpha\left(U^{\prime}\right) \geqslant \alpha(U)$. For the set $U$, we have $x \in U$ and $U \in \mathcal{W}_{k}$. We have shown that the family $\cup_{n=2}^{\infty} \mho_{n}$ covers $X$.

For all $n>1$ and $\alpha<\gamma$, let $V_{\alpha, n}=\bigcup\left\{U \in \mathcal{W}_{n} \mid \alpha(U)=\alpha\right\}$. Further, let $\mathcal{V}_{n}=\left\{V_{\alpha, n} \mid \alpha<\gamma\right\}$ for every $n>1$, and let $\mathcal{V}=\cup_{n=2}^{\infty} \mathcal{V}_{n}$. We have $\cup \mathcal{V}_{n}$ $=\cup \mho_{n}$ for every $n>1$ and hence it follows from the foregoing that the family $\mathcal{V}$ covers $X$. For all $n>1$ and $\alpha<\gamma, V_{\alpha, n}$ is an open set contained in $U_{\alpha}$. It follows that the family $\mathcal{V}$ is an open refinement of $\mathscr{Q}_{1}$. To complete the proof, let $n \in \mathbf{N}, K \subset X$ and $\mathscr{U}^{\prime} \subset \mathscr{Q}_{n}$ be such that the family $\left(\mathscr{U}_{n+1}\right)_{K}$ is a partial refinement of the family $\mathcal{U}^{\prime}$. Let $A=\left\{\alpha\left(U^{\prime}\right) \mid U^{\prime} \in \mathcal{Q}^{\prime}\right\}$ and $B=\{\alpha$ $\left.\langle\gamma| K \cap V_{\alpha, n+1} \neq \varnothing\right\}$. To show that $\left|\left(\mathcal{V}_{n+1}\right)_{K}\right| \leqslant\left|\mathcal{U}^{\prime}\right|$, it suffices to show that $B \subset A$. Let $\beta \in B$. Then we have $K \cap V_{\beta, n+1} \neq \varnothing$ and hence there exists $U \in \mathscr{Q}_{n+1}$ such that $\alpha(U)=\beta$ and $U \cap K \neq \varnothing$. We have $U \in$ $\left(\mathcal{U}_{n+1}\right)_{K}$ and it follows that there is $U^{\prime} \in \mathcal{U}^{\prime}$ such that $U \subset U^{\prime}$. Since $U \in \mathcal{Q} \int_{n+1}$, the family $\mathscr{Q}_{n}$ is precise at $U$ and it follows, since $U \subset U^{\prime} \in$ $\mathcal{U}_{n}$, that $\alpha\left(U^{\prime}\right)=\alpha(U)$. Since $\alpha(U)=\beta$ and $\alpha\left(U^{\prime}\right) \in A$, we have $\beta \in A$.

We have shown that $B \subset A$.

Note that in the above proposition, since $\mathscr{U}_{n+1}$ is a pointwise $W$-refinement of $\mathscr{Q}_{n}$ for every $n \in \mathrm{N}$, each of the families $\mathfrak{V}_{n}$ is point-finite; moreover, if $\mathscr{U}_{n+1}$ is a local $W$-refinement (a local star-refinement) of $\mathscr{U}_{n}$ for every $n \in \mathbf{N}$, then each $\mathcal{V}_{n}$ is locally finite (discrete).

The two results above are the principal tools in this paper for deriving characterizations of metacompactness and paracompactness. However, to be able to use these results, we need another result which shows that for directed interior-preserving open covers, the existence of interior-preserving open star-refinements or $W$-refinements is equivalent with the existence of closurepreserving closed refinements.

A family $\mathcal{L}$ of sets is directed if for all $L \in \mathcal{L}$ and $L^{\prime} \in \mathcal{L}$, there exists $K \in \mathcal{L}$ such that $L \cup L^{\prime} \subset K$. Clearly, every monotone family is directed. If $\mathfrak{T}$ is a family of sets, then we use the symbol $\mathfrak{T}^{F}$ to denote the family consisting of all finite unions of sets from $\mathscr{T}$. Note that $\mathfrak{T}^{F}$ is a directed family and that $\Re$ is directed iff $\Re^{F}$ refines $\Re$. If $\Re$ is an interior-preserving and open (a closure-preserving and closed) cover of $X$, then $\Re^{F}$ is interior-preserving and open (closure-preserving and closed).

LEMMA 2.3. The following conditions are mutually equivalent for an interiorpreserving open cover $\mathcal{Q}$ of $X$ : 
(i) $\mathcal{U}^{F}$ has a closure-preserving closed refinement (by sets whose interiors cover $X$ ).

(ii) $\mathcal{U}^{F}$ has an interior-preserving open pointwise (local) star-refinement.

(iii) थ has an interior-preserving open pointwise (local) $W$-refinement.

Proof. (i) $\Rightarrow$ (iii): Let $\mathscr{F}$ be a closure-preserving closed refinement of $\mathcal{U}^{F}$. For each $x \in X$, let $W(x)=\left[\cap(\mathscr{Q})_{x}\right] \cap\left[X \sim \cup\left(\mathscr{F} \sim(\mathscr{F})_{x}\right)\right]$. The covers $\left\{\cap \mathscr{U}^{\prime} \mid \mathscr{U}^{\prime} \subset \mathscr{U}\right\}$ and $\left\{X \sim \mathcal{U}^{\mathcal{F}^{\prime}} \mid \mathscr{F}^{\prime} \subset \mathscr{F}\right\}$ are interior-preserving and open and it follows that the cover $\mathscr{W}=\{W(x) \mid x \in X\}$ has these same properties. For each $F \in \mathcal{F}$, let $\mathscr{U}(F)$ be a finite subfamily of $\mathscr{U}$ such that $F \subset$ $\cup \mathcal{U}(F)$. We show that for each $F \in \mathscr{F}$, the family $(\mathscr{W})_{F}$ is a partial refinement of the family $\mathscr{U}(F)$. Let $F \in \mathcal{F}$ and let $x \in X$ be such that $W(x) \cap F \neq \varnothing$. From the definition of the set $W(x)$ it follows that $x \in F$. Hence there exists $U \in \mathcal{U}(F)$ such that $x \in U$; for this set $U$ we have $W(x) \subset \cap(\mathcal{Q})_{x} \subset U$. We have shown that $(\mathscr{U})_{F}$ is a partial refinement of $\mathcal{U}(F)$. Since $\mathcal{F}$ is a cover and the families $\mathcal{U}(F), F \in \mathcal{F}$, are finite, it follows from the foregoing that $\mathscr{W}$ is a pointwise $W$-refinement of $\mathscr{Q}$; also, it is clear that if $\{\dot{F} \mid F \in \mathscr{F}\}$ is a cover, then $\mathscr{W}$ is a local $W$-refinement of $\mathscr{U}$.

(iii) $\Rightarrow$ (ii): This is immediate, since any pointwise (local) $W$-refinement of $\mathcal{U}$ is a pointwise (local) star-refinement of $U^{F}$.

(ii) $\Rightarrow$ (i): Let $\mathcal{V}$ be an interior-preserving open pointwise (local) starrefinement of $\mathcal{U}^{F}$. For each $\mathscr{U}^{\prime} \subset \mathcal{U}$, let $F\left(\mathcal{U}^{\prime}\right)=\{x \in X \mid \operatorname{St}(x, \mathfrak{V}) \subset$ $\left.\cup \mathcal{U}^{\prime}\right\}$. Note that if $x \in X$ and $y \in \cap(\mathfrak{V})_{x}$, then $(\mathfrak{V})_{x} \subset(\mathfrak{V})_{y}$ and hence $\operatorname{St}(x, \mathscr{V}) \subset \operatorname{St}(y, \mathcal{V})$. It follows that $x \in F\left(\mathcal{U}^{\prime}\right)$ whenever the set $F\left(\mathcal{U}^{\prime}\right)$ intersects the neighborhood $\cap(\mathscr{V})_{x}$ of $x$. Let $\mathscr{F}=\left\{F\left(\mathcal{U}^{\prime}\right) \mid \mathcal{U}^{\prime} \subset \mathcal{U}\right.$ and $\mathcal{U}^{\prime}$ is finite $\}$. It follows from the foregoing that the family $\mathscr{F}$ is closed and closure-preserving. Since $\mathcal{V}$ is a pointwise (local) star-refinement of $\mathscr{U}$, the family $\mathscr{F}$ (the family $\{\stackrel{\circ}{F} \mid F \in \mathscr{F}\}$ ) covers $X$. Since $F\left(\mathcal{U}^{\prime}\right) \subset \cup \mathcal{U}^{\prime}$ for each $\mathcal{U}^{\prime} \subset \mathcal{U}$, the family $\mathscr{F}$ is a refinement of the family $\mathcal{U}^{F}$.

Since a point-finite open cover is an interior-preserving pointwise $W$ refinement of itself, the above result implies the following [10]: if $\mathscr{U}$ is a point-finite open cover of $X$, then $\mathcal{Q}^{F}$ has a closure-preserving closed refinement.

Considering the above proof, we see that the following holds: an interiorpreserving open cover of a topological space has an interior-preserving open pointwise star-refinement iff the cover has a closure-preserving closed refinement.

3. Interior-preserving covers and covering properties. We start by characterizing metacompact spaces.

THEOREM 3.1. The following conditions are mutually equivalent for a topological space: 
(i) The space is metacompact.

(ii) Every interior-preserving directed open cover of the space has an interiorpreserving open pointwise star-refinement.

(iii) Every interior-preserving directed open cover of the space has a closurepreserving closed refinement.

(iv) Every directed open cover of the space has a closure-preserving closed refinement.

Proof. By the first remark following Lemma 2.3, we have (i) $\Rightarrow$ (iv). That (iv) $\Rightarrow$ (iii) is obvious. The implication (iii) $\Rightarrow$ (ii) is a consequence of Lemma 2.3.

To prove that (ii) $\Rightarrow$ (i), assume that $X$ satisfies condition (ii). To show that $X$ is metacompact, it is enough, by Theorem 2.1 , to show that every interiorpreserving open cover of $X$ has a point-finite open refinement. Let $\mathcal{U}$ be an interior-preserving open cover of $X$. Then the interior-preserving directed open cover $\mathcal{Q}^{F}$ has an interior-preserving open pointwise star-refinement and it follows from Lemma 2.3 that $\mathscr{U}$ has an interior-preserving open pointwise $W$-refinement $\mathscr{Q}_{2}$. Using induction, we see that there exists a sequence $\left\langle\mathscr{U}_{n}\right\rangle$ of interior-preserving open covers of $X$ such that $\mathscr{Q}_{1}=\mathscr{U}$ and for each $n \in \mathbf{N}$, the cover $\mathscr{U}_{n+1}$ is a pointwise $W$-refinement of the cover $\mathscr{U}_{n}$. Using Proposition 2.2 and the remark made after that proposition, we see that $\mathscr{Q}$ has an open refinement $\cup_{n \in \mathrm{N}} \mho_{n}$ such that each $\mho_{n}$ is point-finite. For each $n \in \mathbf{N}$, let $V_{n}=\cup\left\{W \mid W \in \cup_{k=1}^{n} \mho_{n}\right\}$. Then the family $\mathscr{V}=\left\{V_{n} \mid n \in \mathbf{N}\right\}$ is an interior-preserving directed open cover of $X$. Let $\mathscr{P}$ be an open pointwise star-refinement of $\mathcal{V}$. Let $F_{0}=\varnothing$ and for each $n \in \mathbf{N}$, let $F_{n}=\{x$ $\left.\in X \mid \operatorname{St}(x, \mathcal{P}) \subset V_{n}\right\}$; note that the sets $F_{n}$ are closed. Let $\mathscr{W}_{n}^{\prime}=\{W \sim$ $\left.F_{n-1} \mid W \in \mho_{n}\right\}$ for each $n \in \mathbf{N}$. It is easily seen that the family $\mathscr{W}=$ $\cup_{n \in N} W_{n}^{\prime}$ is a point-finite open cover of $X$; moreover, the cover $W$ is a refinement of the cover $\mathcal{U}$.

COROLlary 3.2 [23]. The continuous image of a metacompact space under a closed mapping is metacompact.

Proof. Let $X$ be a metacompact space and let $f: X \rightarrow Y$ be a closed and continuous mapping from $X$ onto a topological space $Y$. To show that $Y$ is metacompact, let $\mathscr{Q}$ be a directed open cover of $Y$. Then the family $\mathcal{V}=\left\{f^{-1}(U) \mid U \in \mathcal{Q}\right\}$ is a directed open cover of $X$. By Theorem 3.1, the cover $\mathcal{W}$ has a closure-preserving closed refinement, say $\mathscr{F}$. It is easily seen that the family $\{f(F) \mid F \in \mathscr{F}\}$ is a closure-preserving closed refinement of थ. The conclusion now follows from Theorem 3.1.

Since a cover by compact subsets of $X$ is a refinement of every directed open cover of $X$, the following result is a direct consequence of Theorem 3.1. 
Corollary 3.3 ([11] and [15]). A topological space is metacompact if the space has a closure-preserving cover by compact, closed subsets.

We turn to consider paracompact spaces.

THEOREM 3.4. The following conditions are mutually equivalent for a topological space:

(i) The space is paracompact.

(ii) Every interior-preserving directed open cover of the space has an interiorpreserving open local star-refinement.

(iii) Every interior-preserving directed open cover of the space has a oclosure-preserving refinement by closed sets whose interiors cover the space.

(iv) Every directed open cover of the space has a closure-preserving refinement by closed sets whose interiors cover the space.

Proof. Since a locally finite open cover is a local $W$-refinement of itself, it follows from Lemma 2.3 that (i) $\Rightarrow$ (iv). That (iv) $\Rightarrow$ (iii) is obvious.

(iii) $\Rightarrow$ (ii): Assume that $X$ satisfies condition (iii). To show that $X$ satisfies condition (ii), we show first that $X$ is countably paracompact. Let $\mathcal{C}=\left\{C_{n} \mid n\right.$ $\in \mathbf{N}\}$ be a countable open cover of $X$. For each $n \in \mathbf{N}$, let $D_{n}=\cup_{k=1}^{n} C_{k}$. Then the family $\mathscr{D}=\left\{D_{n} \mid n \in \mathbf{N}\right\}$ is an interior-preserving directed open cover of $X$. Let $\mathcal{S}=\cup_{n \in N} \delta_{n}$ be a closed refinement of $\mathscr{D}$ such that the family $\{\dot{S} \mid S \in \mathcal{S}\}$ covers $X$ and for each $n \in N$, the family $\mathcal{S}_{n}$ is closurepreserving. Let $R_{0}=\varnothing$ and for each $n \in \mathbf{N}$, let $R_{n}=\cup\left\{S \in \cup_{k=1}^{n} \delta_{n} \mid S \subset\right.$ $\left.D_{n}\right\}$; then it is easily seen that the family $\left\{C_{n} \sim R_{n-1} \mid n \in \mathbf{N}\right\}$ is a locally finite open refinement of the cover $C$.

Next we show that if $\mathcal{F}$ is a $\sigma$-closure-preserving family of closed subsets of $X$ such that the sets $\dot{F}, F \in \mathscr{F}$, cover $X$, then $\mathscr{F}$ has a closure-preserving closed refinement $\mathcal{K}$ such that the sets $\dot{K}, K \in \mathcal{K}$, cover $X$. Let $\mathcal{F}=$ $\cup_{n \in N} \mathscr{F}_{n}$ be a family of closed subsets of $X$ such that $\{\dot{F} \mid F \in \mathscr{F}\}$ is a cover of $X$ and for each $n \in \mathbf{N}$, the family $\mathscr{F}_{n}$ is closure-preserving. For each $n \in \mathbf{N}$, let $C_{n}=\cup\left\{\stackrel{F}{F} \mid F \in \mathscr{F}_{n}\right\}$. Then the family $\mathcal{C}=\left\{C_{n} \mid n \in \mathbf{N}\right\}$ is a countable open cover of $X$ and it follows from the foregoing that $\mathcal{C}$ has a locally finite open refinement, say $\mathcal{P}$. For each $P \in \mathscr{P}$, let $n(P) \in \mathbf{N}$ be such that $P \subset C_{n(P)}$ and let $\mathcal{K}(P)=\left\{F \cap \bar{P} \mid F \in \mathscr{F}_{n(P)}\right\}$. Then the family $\mathcal{K}=$ $\cup\{\mathfrak{K}(P) \mid P \in \mathscr{P}\}$ is a closed refinement of $\mathscr{F}$. For each $P \in \mathscr{P}$, the family $\mathcal{K}(P)$ is closure-preserving and it follows, since $\mathcal{P}$ is locally finite, that the family $\mathscr{K}$ is closure-preserving. Moreover, if $x \in X$ and if $P \in(\mathscr{P})_{x}$, then we have $x \in \stackrel{K}{K}$ for some $K \in \mathscr{K}(P)$; it follows that the family $\{\stackrel{K}{K} \mid K \in \mathscr{K}\}$ covers $X$.

It follows from the foregoing that every interior-preserving directed open cover of $X$ has a closure-preserving refinement by closed sets whose interiors cover $X$. Using the result of Lemma 2.3, we see that $X$ satisfies condition (ii). 
(ii) $\Rightarrow$ (i): Assume that $X$ satisfies condition (ii). Note that it follows from Lemma 2.3 that $X$ also satisfies condition (iii); hence, by the preceding part of this proof, $X$ is countably paracompact. To show that $X$ is paracompact, it is enough, by Theorem 2.1, to show that every interior-preserving open cover of $X$ has a locally finite open refinement. Let $\mathscr{Q}$ be an interior-preserving open cover of $X$. Substituting "local" for "pointwise" in the corresponding part of the proof of Theorem 3.1, we see that, in the case at hand, $\mathscr{U}$ has an open refinement $\cup_{n \in \mathrm{N}} \mathscr{W}_{n}$ such that each $\mathscr{W}_{n}$ is locally finite. For each $n \in \mathbf{N}$, let $V_{n}=\cup \mathcal{W}_{n}$. Since $X$ is countably paracompact, the countable open cover $\left\{V_{n} \mid n \in \mathbf{N}\right\}$ of $X$ has a locally finite open refinement $\mathcal{P}$. For each $P \in \mathcal{P}$, let $n(P) \in \mathrm{N}$ be such that $P \subset V_{n(P)}$ and let $\mathcal{L}(P)=\left\{W \cap P \mid W \in \mathcal{W}_{n(P)}\right\}$. Then the family $\mathcal{Q}=\cup\{\mathcal{Q}(P) \mid P \in \mathscr{P}\}$ is a locally finite open refinement of थ.

In [12], J. Mack proved that a topological space is paracompact iff every directed open cover of the space has a locally finite closed refinement. If $\mathscr{K}$ is a locally finite closed cover of $X$, then the family $\mathcal{K}^{F}$ is closure-preserving and closed and the sets $\dot{S}, S \in \mathcal{K}^{F}$, cover $X$. Hence the sufficiency of Mack's condition for paracompactness can be derived using the above theorem.

For regular spaces, we have the following corollary to Theorem 3.4:

COROLlaRY 3.5. A regular space is paracompact iff every directed open cover of the space has a $\sigma$-closure-preserving open refinement.

Proof. Since locally finite families are closure-preserving, the condition is necessary. To prove sufficiency, let $X$ be a regular space and let $\mathscr{U}$ be a directed open cover of $X$. Then there exists an open cover $\mathcal{V}$ of $X$ such that the family $\{\bar{V} \mid V \in \mathcal{V}\}$ refines $\mathcal{U}$. Since $\mathcal{U}$ is directed, we see that the family $\left\{\bar{W} \mid W \in \mathcal{V}^{F}\right\}$ refines $\mathcal{Q}$. If the directed open cover $\mathcal{F}^{F}$ has a $\sigma$-closurepreserving open refinement 2 , then the family $\{\bar{Q} \mid Q \in \mathcal{Q}\}$ is a $\sigma$-closurepreserving refinement of $\mathscr{U}$ by closed sets whose interiors cover $X$. This observation and the result of Theorem 3.4 show that $X$ is paracompact if every directed open cover of $X$ has a $\sigma$-closure-preserving open refinement.

The last result of this section shows what happens if we delete the word "directed" from condition (iii) of Theorem 3.1.

THEOREM 3.6. A topological space is paracompact if every interior-preserving open cover of the space has a closure-preserving closed refinement.

Proof. Assume that $X$ satisfies the condition stated in the theorem. Then it follows from Theorem 3.1 that $X$ is metacompact. We now use Theorem 3.4 to show that $X$ is paracompact. Let $\mathcal{U}$ be a directed open cover of $X$. Since $X$ is metacompact, there exists a point-finite open cover $\mathfrak{V}$ of $X$ such that $\mathfrak{V}$ 
refines $\mathcal{U}$. Since $\mathfrak{V}$ is interior-preserving it has a closure-preserving closed refinement, say $\mathscr{F}$. For each $V \in \mathcal{V}$, let $K(V)=\cup\{F \in \mathscr{F} \mid F \subset V\}$. Since the family $\mathcal{V}$ is point-finite, the family $\mathcal{K}=\{K(V) \mid V \in \mathcal{V}\}$ has this same property. The family $\mathcal{K}$ is also closure-preserving and closed and it follows that $\mathcal{K}$ is locally finite; since $\mathscr{K}$ is a cover, it follows further that the sets $N$, $N \in \mathscr{K}^{F}$, cover $X$. The family $\mathscr{K}^{F}$ is a closure-preserving refinement of the directed cover $\mathcal{U}$ by closed sets whose interiors cover $X$. The conclusion now follows from Theorem 3.4.

Note that the above theorem can also be proved using the second remark following Lemma 2.3 together with well-known results concerning normal sequences of coverings (see [21] and [19]).

Leaving out the word "directed" from Corollary 3.5 and "interior-preserving" from Theorem 3.6, we have two results of E. Michael [13].

4. Some applications. We now apply the results of the preceding section to prove some further results concerning metacompact and paracompact spaces.

A topological space is orthocompact (see [8]) if every open cover of the space has an interior-preserving open refinement. By a result in [7], every linearly ordered topological space is orthocompact; hence we see that orthocompactness is a strictly weaker property than metacompactness. Nevertheless, for subspaces of regular compact spaces, we can characterize metacompactness in terms of orthocompactness as follows.

THEOREM 4.1. A subspace $X$ of a compact and regular space $K$ is metacompact iff the product space $K \times X$ is orthocompact.

Proof. Since every metacompact space is orthocompact, necessity of the condition follows from the result that the product of a metacompact space with a compact space is metacompact (see [6, Exercise 5.3.H]).

Sufficiency. Let $K$ be a regular and compact space and let $X$ be a subspace of $K$ such that the space $K \times X$ is orthocompact. We use Theorem 3.1 to show that $X$ is metacompact. Let $\mathcal{Q}$ be a directed open cover of $X$. Since $X$ is a subspace of $K$, there exists a family $\mathfrak{V}$ of open subsets of $K$ such that $\mathcal{Q}=\{V \cap X \mid V \in \mathcal{V}\}$. Denote the set $\cup \mathcal{V}$ by $O$. Since $K$ is a regular space, there exists a family $\mho$ of open subsets of $K$ such that $\cup \mho=O$ and $\bar{W} \subset O$ for each $W \in \mathcal{W}$. It is easily seen that the family $\theta=\{O \times X\} \cup$ $\{(K \sim \bar{W}) \times(W \cap X) \mid W \in \mathcal{W}\}$ is an open cover of the product space $K \times X$. Let $\mathscr{P}$ be an interior-preserving open cover of $K \times X$ such that $\mathcal{P}$ refines $\mathcal{O}$. The family $R=\left\{\cup^{\mathcal{P}^{\prime}} \mid \mathcal{P}^{\prime} \subset \mathcal{P}\right\}$ is interior-preserving and open; hence the family $\mathscr{F}=\{(K \times X) \sim R \mid R \in \Re\}$ is closure-preserving and closed. Since $K$ is a compact space, the projection map $p: K \times X \rightarrow X$ is closed (see e.g. [6, Theorem 3.1.6]). It follows that the family $\mathcal{K}=\{p(F) \mid F$ $\in \mathscr{F}\}$ of subsets of the space $X$ is closure-preserving and closed. If we let 
$K(x)=\cap(\mathcal{H})_{x}$ for every $x \in X$, then the family $\mathscr{K}=\{K(x) \mid x \in X\}$ is closure-preserving and closed.

We show that $\mathscr{K}$ is a refinement of $\mathcal{Q}$. Since $x \in K(x)$ for each $x \in X$ (we have $K(x)=X$ if $\left.(\mathcal{C})_{x}=\varnothing\right)$, it suffices to show that $\mathcal{K}$ is a partial refinement of $\mathscr{Q}$. Let $x \in X$. If we set $N(x)=\cup\left\{\cap(\mathscr{P})_{(k, x)} \mid k \in K \sim O\right\}$, then $N(x)$ is open in $K \times H$ and it follows that the set $L(x)=\{h \in K \mid(h, x) \notin$ $N(x)\}$ is a closed, and hence compact, subset of $K$. We clearly have $L(x) \subset O$ and it follows that $L(x) \subset G$ for some $G \in \mathcal{F}$; since $\mathscr{U}=\{V \cap X \mid V \in$ $\mathscr{V}\}$ and $\mathscr{Q}$ is a directed family, it follows that $L(x) \cap X \subset U$ for some $U \in \mathcal{Q}$. Hence to show that $K(x) \subset U$ for some $U \in \mathcal{Q}$, it suffices to show that $K(x) \subset L(x)$. Assume on the contrary that $K(x) \not L L(x)$ and let $y \in$ $K(x) \sim L(x)$. Since $y \notin L(x)$, we have $(y, x) \in N(x)$ and hence there exists $k \in K \sim O$ such that $(y, x) \in \cap(\mathscr{P})_{(k, x)}$. Let $T=(K \times X) \sim \operatorname{St}(K \times$ $\{y\}, \mathscr{P})$. Then we have $T \in \mathcal{F}$ and $y \notin p(T)$ and it follows, since $y \in K(x)$, that $x \notin p(T)$. Hence we have $K \times\{x\} \subset \operatorname{St}(K \times\{y\}, \mathcal{P})$. It follows that there exist $h \in K$ and $P \in \mathscr{P}$ such that $\{(k, x),(h, y)\} \subset P$. Since $(y, x) \in$ $\cap(\mathscr{P})_{(k, x)}$, we also have $(y, x) \in P$. We have $P \not \subset O \times X$, because $k \notin O$, and it follows, since $\mathcal{P}$ refines $\mathcal{O}$, that there exists $W \in \mathcal{W}$ such that $P \subset(K \sim \bar{W}) \times(W \cap X)$. But then we have $\{(y, x),(h, y)\} \subset(K \sim \bar{W}) \times$ $(W \cap X)$ and this is impossible. It follows that $K(x) \subset L(x)$ and hence that $K(x) \subset U$ for some $U \in \mathcal{Q}$.

We have shown that every directed open cover of $X$ has a closure-preserving closed refinement. By Theorem 3.1, $X$ is metacompact.

In [17], B. M. Scott has shown that several theorems concerning normality of product spaces with paracompact factors remain true if one replaces "paracompact" by "metacompact" and "normal" by "orthocompact" in the theorems. H. Tamano proved in [20] that a Tychonoff-space $X$ is paracompact iff the product space $X \times \beta X$ is normal. If $X$ is a Tychonoff-space and if we take $K=\beta X$ in the above theorem, then we obtain the following analogue of Tamano's theorem.

Corollary 4.2. A Tychonoff-space $X$ is metacompact iff the product space $X \times \beta X$ is orthocompact.

An open neighbornet of a topological space $X$ is a binary relation $V$ on $X$ such that for each $x \in X$, the set $V\{x\}$ is an open neighborhood of $x$ (see [10]); we say that $V$ is co-compact if for each $x \in X$, the set $V^{-1}\{x\}$ is compact. If $\mathscr{F}$ is a closure-preserving closed cover of $X$ and if we define a relation $V$ on $X$ by setting $V\{x\}=X \sim U\left(\mathscr{F} \sim(\mathscr{F})_{x}\right)$ for each $x \in X$, then $V$ is an open neighbornet of $X$ and we have $V^{-1}\{x\}=\cap(\mathscr{F})_{x}$ for each $x \in X$ (see [10, Theorem 3.14]); if the sets in $\mathscr{F}$ are compact, then $V$ is co-compact. Hence the following improves the result mentioned in Corollary 3.3 above. 
THEOREM 4.3. A topological space is metacompact if the space has a co-compact open neighbornet.

Proof. Let $V$ be a co-compact open neighbornet of $X$. We show that every interior-preserving directed open cover of $X$ has a closure-preserving closed refinement; it then follows from Theorem 3.1 that $X$ is metacompact. Let $\mathscr{Q}$ be an interior-preserving directed open cover of $X$. We define an open neighbornet $W$ on $X$ by setting $W\{x\}=\cap(U)_{x}$ for each $x \in X$. For every $x \in X$, we have $W^{-1}\{x\}=X \sim \cup\left(U \sim(U)_{x}\right)$ and hence the set $W^{-1}\{x\}$ is closed. It follows that the relation $N=V \cap W$ is a co-compact open neighbornet. For each $U \in \mathcal{Q}$, the set $F(U)=\left\{x \in X \mid N^{-1}\{x\} \subset U\right\}$ is closed and we have $F(U) \subset U$. Since $\mathcal{Q}$ is a directed open cover and the neighbornet $N$ is co-compact, the family $\mathcal{F}=\{F(U) \mid U \in \mathcal{U}\}$ covers $X$. It follows that $\mathcal{F}$ is a closed refinement of $\mathscr{U}$. To show that $\mathscr{F}$ is closurepreserving, let $x \in X$. Since the open family $\left\{N\{y\} \mid y \in N^{-1}\{x\}\right\}$ covers the compact set $N^{-1}\{x\}$, there is a finite subset $A$ of $N^{-1}\{x\}$ such that $N^{-1}\{x\} \subset N A$. Denote by $Q$ the neighborhood $\cap\{N\{a\} \mid a \in A\}$ of $x$. Let $U \in \mathcal{Q}$ be such that $F(U) \cap Q \neq \varnothing$. We show that $x \in F(U)$. Let $z \in$ $F(U) \cap Q$. Then we have $A \subset N^{-1}\{z\} \subset U$. For each $a \in A$, we have $N\{a\} \subset \cap(\mathscr{U})_{a} ;$ since $A \subset U$, it follows that $N A \subset U$. Since $N^{-1}\{x\} \subset$ $N A$, it follows that $N^{-1}\{x\} \subset U$, in other words, that $x \in F(U)$. We have shown that if $U \in \mathcal{Q}$ and $F(U) \cap Q \neq \varnothing$, then $x \in F(U)$. It follows from the foregoing that the family $\mathscr{F}$ is closure-preserving.

We need two definitions to state our last result. $X$ is isocompact [3] if every countably compact closed subspace of $X$ is compact. $X$ is an $M^{\#-s p a c e ~[18] ~ i f ~}$ $X$ has a sequence $\left\langle\mathcal{K}_{n}\right\rangle$ of closure-preserving closed covers such that the following condition is satisfied:

(M) if $x \in X$ and if $\left\langle A_{n}\right\rangle$ is a decreasing sequence of nonempty sets such that $A_{n} \subset \operatorname{St}\left(x, \mathcal{K}_{n}\right)$ for each $n \in \mathrm{N}$, then $\bigcap_{n \in \mathrm{N}} \bar{A}_{n} \neq \varnothing$.

\section{THEOREM 4.4. An isocompact $M^{\#-s p a c e ~ i s ~ p a r a c o m p a c t . ~}$}

Proof. Let $X$ be an isocompact $M^{\sharp}$-space and let $\left\langle\mathcal{K}_{n}\right\rangle$ be a sequence of closure-preserving closed covers of $X$ such that $\left\langle\mathcal{K}_{n}\right\rangle$ satisfies $(M)$. We may assume that for each $n \in \mathbf{N}, \mathscr{K}_{n+1}$ is a refinement of $\mathscr{K}_{n}$. For each $x \in X$, denote by $K(x)$ the closed set $\bigcap_{n \in N} \operatorname{St}\left(x, \mathcal{K}_{n}\right)$. It follows from Proposition 1.6 of [14], that for each $x \in X$, the set $K(x)$ is countably compact and the sequence $\left\langle\operatorname{St}\left(x, \mathcal{K}_{n}\right)\right\rangle$ is a network at this set. Since $X$ is isocompact, the sets $K(x), x \in X$, are compact.

We use Theorem 3.4 to show that $X$ is paracompact. Let $\mathcal{Q}$ be a directed open cover of $X$. For each $x \in X$, let $U(x) \in \mathcal{U}$ be such that the compact set $K(x)$ is contained in the set $U(x)$. Then, for each $x \in X$, there exists $n(x) \in \mathbf{N}$ such that the set $\operatorname{St}\left(x, \mathscr{K}_{n(x)}\right)$ is contained in the neighborhood 
$U(x)$ of the set $K(x)$. For every $n \in \mathbf{N}$, denote by $\mathscr{F}_{n}$ the family $\left\{\operatorname{St}\left(x, \mathcal{K}_{n}\right) \mid x\right.$ $\in X$ and $n(x)=n$ \} and note that this family is closure-preserving and closed since $\mathscr{F}_{n}$ has these properties. The family $\mathscr{F}=\bigcup_{n \in \mathscr{X}} \mathscr{F}_{n}$ is a $\sigma$-closurepreserving closed refinement of $\mathcal{Q}$ and the family $\{\stackrel{\circ}{F} \mid F \in \mathscr{F}\}$ covers $X$, since we have $x \in \operatorname{Int}\left(\operatorname{St}\left(x, \mathcal{K}_{n(x)}\right)\right)$ for every $x \in X$. We have shown that $X$ satisfies condition (iv) of Theorem 3.4; it follows that $X$ is paracompact.

By a result in [2], every metacompact space is isocompact; hence it follows from the theorem above that isocompactness is equivalent with paracompactness in the class of $M^{\#-s p a c e s . ~ I n ~[5] ~ i t ~ i s ~ s h o w n ~ t h a t ~ a ~ c o v e r i n g ~ p r o p e r t y ~}$ much weaker than metacompactness implies isocompactness and by a result in [4], every topological space with a $G_{\delta}$-diagonal is isocompact; since every paracompact, Hausdorff, $M^{\#}$-space is an $M$-space (this follows from Theorem 2.3 of [9]), these results and the above theorem show that many of the metrization theorems involving $M$-spaces remain true for the larger class of $M^{\#}$-spaces.

Acknowledgement. The author is grateful to Professor Peter Fletcher for his helpful advise and comments.

\section{REFERENCES}

1. P. Alexandroff and P. Urysohn, Mémoire sur les espaces topologiques compacts, Verh. Nederl. Akad. Wetensch. (Amsterdam) 14 (1929), 1-96.

2. R. Arens and J. Dugundji, Remark on the concept of compactness, Portugal. Math. 9 (1950), 141-143.

3. P. Bacon, The compactness of countably compact spaces, Pacific J. Math. 32 (1970), 587-592.

4. J. Chaber, Conditions which imply compactness in countably compact spaces, Bull. Acad. Polon. Sci. Sér. Sci. Math. Astronom. Phys. 24 (1976), 993-998.

5. S. W. Davis, A cushioning-type weak covering property, Pacific J. Math. (to appear).

6. R. Engelking, General topology, PWN-Polish Scientific Publishers, Warszawa, 1977.

7. W. M. Fleischman, On fundamental open coverings, in: D. R. Kurepa, ed., Proceedings of the International Symposium on Topology, and its Applications, Herzeg-Novi, 25.-3.1.8, 1968, Yugoslavia, Savez Drustava Mat. Fiz. Astron., Belgrade, 1969, pp. 154-155.

8. P. Fletcher and W. F. Lindgren, Transitive quasi-uniformities, J. Math. Anal. Appl. 39 (1972), 397-405.

9. T. Ishii, On wM-spaces. I, Proc. Japan Acad. 46 (1970), 5-10.

10. H. J. K. Junnila, Neighbornets, Pacific J. Math. 76 (1978), 83-108.

11. Y. Katuta, On spaces which admit closure-preserving covers by compact sets, Proc. Japan Acad. 50 (1974), 826-828.

12. J. Mack, Directed covers and paracompact spaces, Canad. J. Math. 19 (1967), 649-654.

13. E. Michael, Another note on paracompact spaces, Proc. Amer. Math. Soc. 8 (1957), 822-828.

14. , A quintuple quotient quest, General Topology and Appl. 2 (1972), 91-138.

15. H. Potoczny and H. Junnila, Closure-preserving families and metacompactness, Proc. Amer. Math. Soc. 53 (1975), 523-529.

16. W. B. Sconyers, Metacompact spaces and well-ordered open coverings, Notices Amer. Math. Soc. 18 (1971), 230.

17. B. M. Scott, Toward a product theory of orthocompactness, N. M. Stavrakas and K. R. Allen, eds., Studies in Topology, Academic Press, New York, 1975, pp. 517-537.

18. F. Siwiec and J. Nagata, $A$ note on nets and metrization, Proc. Japan Acad. 44 (1968), 623-627. 
19. A. H. Stone, Paracompactmess and product spaces, Bull. Amer. Math. Soc. 54 (1948), 977-982.

20. H. Tamano, On paracompactness, Pacific J. Math. 10 (1960), 1043-1047.

21. J. W. Tukey, Convergence and uniformity in topology, Princeton Univ. Press, Princeton, N.J., 1940.

22. J. M. Worrell, Jr., A characterization of metacompact spaces, Portugal. Math. 25 (1966), 171-174.

23.

The closed contimuous images of metacompact topological spaces, Portugal. Math. 25 (1966), 175-179.

Departmient of mathematics, Virgnia Polytbchinic Institute and State University, Blacksburg, Virginia 24060

Current address: Department of Mathematics, University of Pittsburgh, Pittsburgh, Pennsylvania 15260 